\title{
Paper Battery Available Capacity Meter Built into an AC/DC Telecom Power Supply System
}

\author{
Paweł Godlewski, Ryszard Kobus, and Paweł Kliś \\ National Institute of Telecommunications, Warsaw, Poland
}

\begin{abstract}
The article describes the results of testing a Benning AC/DC power supply, with integrated NIT TBA-ST meter. Such integration enables accurate and energy-efficient measurements of available capacity of individual 48-voltage VRLA/AGM batteries with remote management possibilities. The full compliance with the requirements for telecommunication power systems, the great functionality, upgrade ability, immunity to user errors and the high accuracy of capacity and voltage measurement of battery monoblocks of proposed solution are presented.
\end{abstract}

Keywords-battery capacity tester, battery discharge tester, VRLA real capacity.

\section{Introduction}

In $\mathrm{AC} / \mathrm{DC}$ power supply systems, during voltage interruption energy backup is provided by lead acid batteries, mainly of the VRLA/AGM type. They are inexpensive, safe and their high fault current enables fast tripping of fuses. However, owing to certain unfavorable phenomena, i.e. premature capacity loss (PCL), VRLA/AGM batteries require periodical capacity testing.

The most commonly operated telecommunication power systems (Fig. 1a), contain at least two 48-voltage batteries B connected by a common disconnector switch RGR, system bus with + on the ground with rectifier outputs PS and DC load R. The operation is managed by the ST controller, which also ensures communication with the SN supervisor center. Under normal operation conditions, the rectifiers feed load $\mathrm{R}$ and charge batteries keeping them fully charged.

When AC mains failure occurs the power supply to load is taken from batteries, and after voltage is restored, rectifiers again feed load and charge batteries. The circuit also has a common high-current disconnector switch RGR, which protects batteries from deep discharging. It breaks the circuit when battery voltage drops below $40 \mathrm{~V}$ in the long absence AC mains network. In such a power system, the measurement of available capacity of any battery can be performed by portable instruments, e.g. RRR shown in Fig. 1a, or electronic loads as well as TBA-IŁ [1]. The measurements require disconnection of the battery from the system bus by service operator. While testing, the necessary energy reserve is provided by the other batteries.
In AC/DC power systems with TBA-ST meter built-in (Fig. 1b), the RGR disconnector switch and PN low-current contactor, are connected in series with each battery Bn [2], and to the meter output. First, the instrument discharges the battery for test purposes, next recharges it and calculates the capacity. After that the battery is connected back to the system bus and capacity data is transferred to $\mathrm{SN}$.

\section{TBA-ST Unit}

The TBA-ST meter has been designed and made at the National Institute of Telecommunications under the energy reserve checking system for telecommunication sites project (SKOT), co-financed by the European Union with European Regional Development Funds (ERDF). The meter occupies half of the 19"/1U rack frame (Fig. 2a).

The circuit is based on a bi-directional boost-buck DC/DC converter allowing increase or decrease input voltage, with control circuit and voltage and current sensors (Fig. 2). The converter can deliver up to $50 \mathrm{~A}$ output current and operates similarly to the TBA-IL devices. By battery discharging with preset current, it transfers energy to the system bus, which temporarily sheds load from the rectifiers. By charging the battery with preset current to a programmed voltage, the unit draws energy from system bus and continuously measures voltage on the power system and the battery under test, as well as voltages of monoblocks of all present system batteries. The "LEM" current transducer allows battery current measurement and thus capacity. The configuration parameters and measurement results are stored in controller non-volatile memory. The communication with the power system controller is DC-based, and data exchange with the SN takes place via the RS485 and the ZSN-5 communication module of the SCS Win system or by a built-in Ethernet interface.

\subsection{Operation}

The battery test is initiated remotely the controller SN. First the TBA-ST unit checks if the test can be performed. If yes, it sends a command to the power system controller. Test begins after battery reconnecting to the test unit. The discharging phase ends when a predefined charge (Ah) has been sink and voltage have been reduced on bat- 
(a)

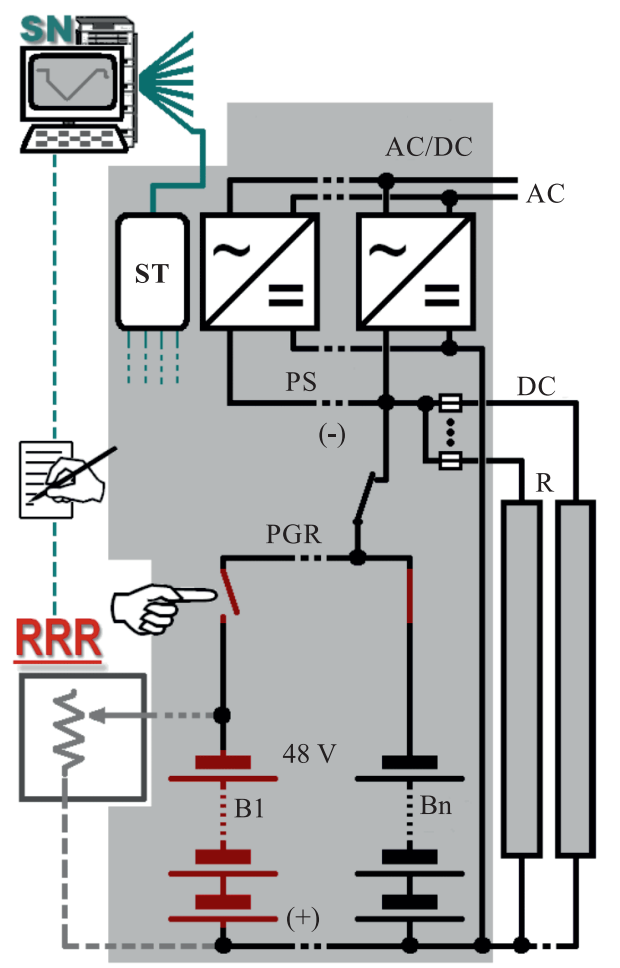

(b)

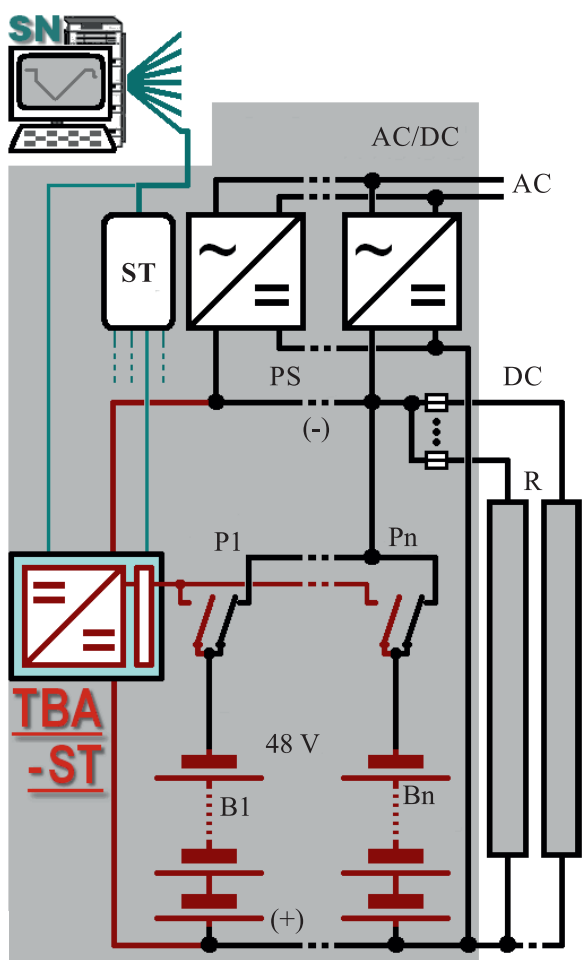

Fig. 1. AC/DC power system with battery capacity meter: (a) as portable "RRR" instrument and (b) as built-in "TBA-ST" unit.

(a)

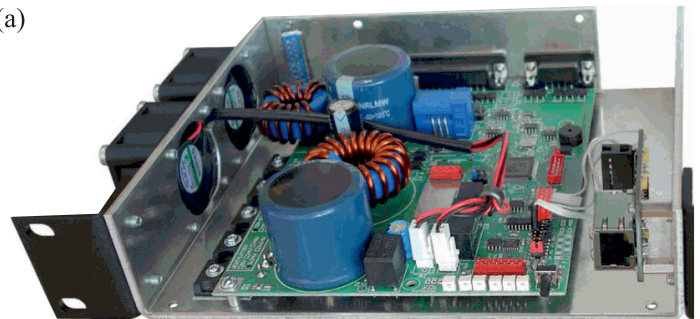

(b)

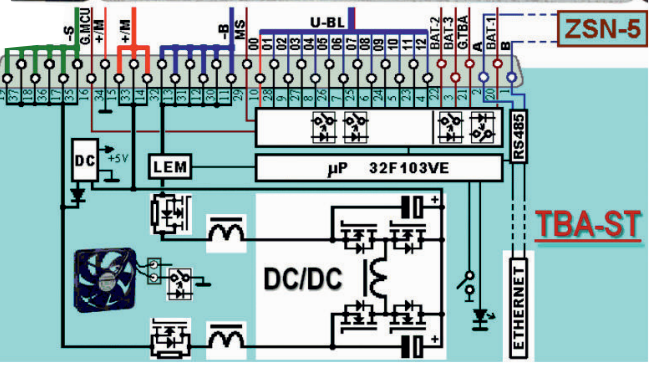

Fig. 2. VRLA battery capacity meter TBA-ST: (a) overview, (b) block diagram.

tery monoblocks to a programmed value. Charging phase takes place for given time calculated from the moment the battery voltage preset is reached. The test is ended with battery reconnecting. An initiated test can be remotely canceled or break with a stop button. The cycle is then interrupted, the battery is fully charged and reconnected to the power system as soon as possible.

\section{AC/DC Power System}

In conducted measurements the Benning AC/DC power supply system "SBE200SL version SKOT" was used. It was configured in accordance with requirements received from the National Institute of Telecommunications for operation with the TBA-ST. The solution is protected by a European patent of the National Institute of Telecommunications.

The system was housed in a PSJ1866 $600 \times 600 \times 1870 \mathrm{~mm}$ cabinet (Fig. 3a). It contains three $48 \mathrm{~V} / 1500 \mathrm{~W}$ rectifier modules (PS) with AC current protection devices, a distribution panel for 15 loads with DC current protection devices $(F)$, a current protection supervisor card, three high current disconnectors and three contactors (P). MCU2500 controller (ST), a signaling module, two input/output cards (IO), three $48 \mathrm{~V} / 100 \mathrm{Ah}(4 \times 12 \mathrm{~V})$ battery strings $(\mathrm{B})$, as well as connectors. There is a free space for the TBA-ST meter and ZSN-5 communication module in enclosure.

The block diagram shown in Fig. $3 b$ presents the TBAST meter circuit. The contact components (switches and contactors) are shown in which battery B1 is tested. The power system ensures automatic operation with temperature compensation of floating voltage and programmable battery charging current (via resistor R1). The standard power system operation program featuring macro instructions of non-standard use of the inputs/outputs of the IO/02 card. The power system was not equipped with battery voltage measurement cards and thus the battery "auto test" func- 
(a)

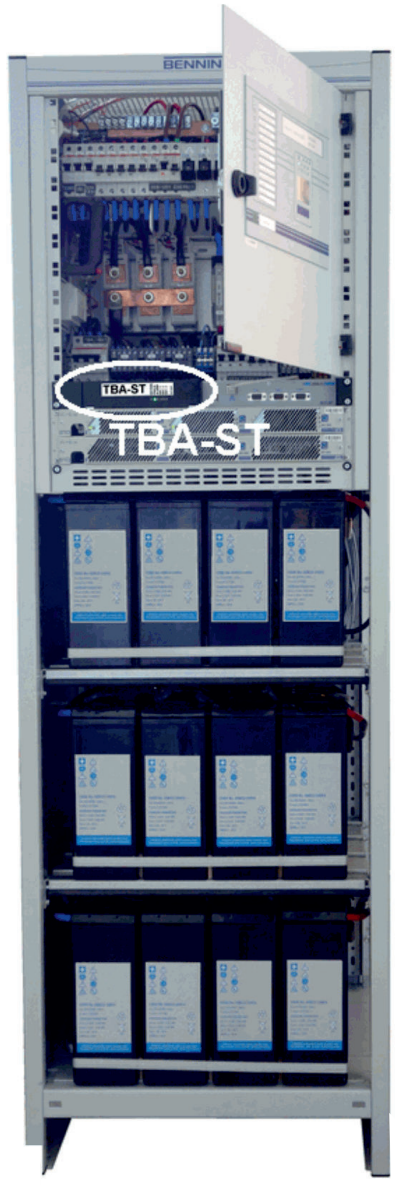

(b)

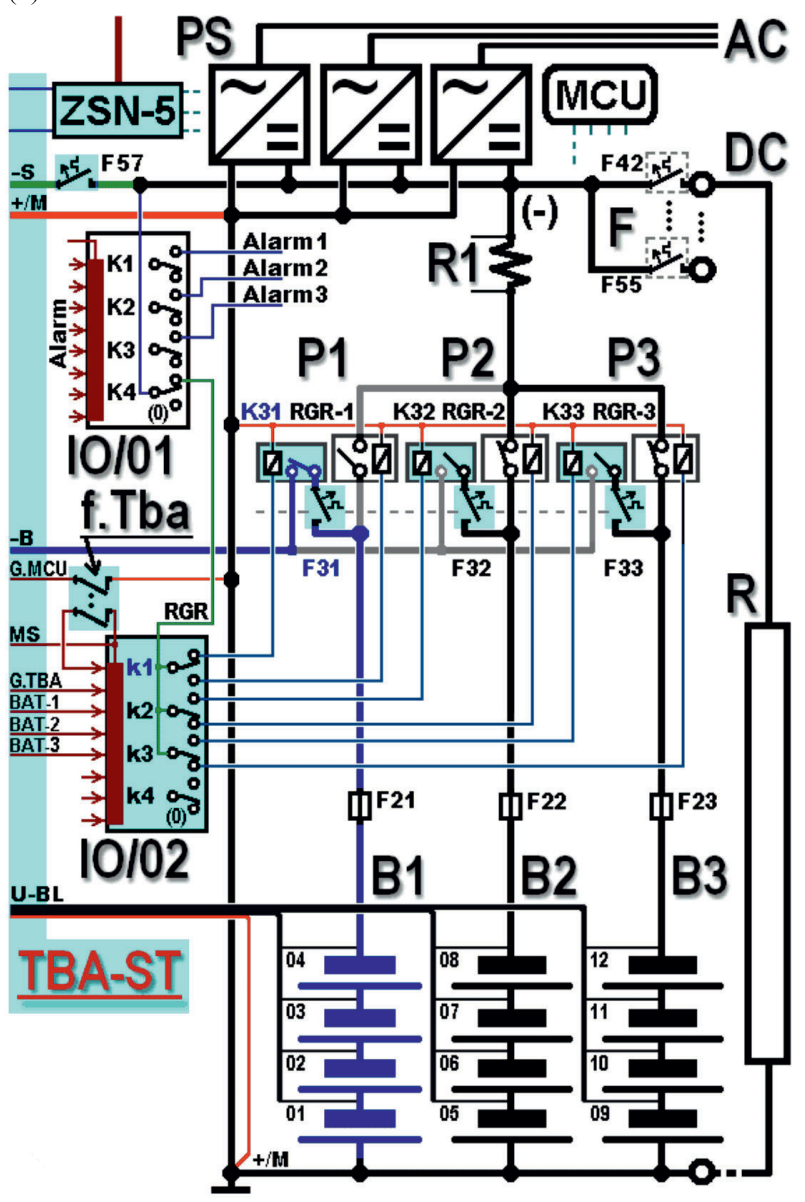

Fig. 3. AC/DC power system equipped with $3 \times 48 \mathrm{~V} / 100$ Ah batteries and modified to use TBA-ST unit: (a) overview, (b) block diagram.

tion was not checked, because such measurements are performed with greater accuracy by the TBA-ST meter. During testing, worn battery banks (HZB12-100FA) were used, as this reduced the time of energy reserve, and consequently the time of testing the individual functions.

\subsection{Operation without the TBA-ST}

If the circuit breaker f.Tba is open, the power system operates in standard mode [3] with the one difference that three RGR disconnectors are used instead of one. They are controlled by the $\mathrm{K} 4$ relay located on the $\mathrm{IO} / 01$ card. The relay contactors $\mathrm{k} 1, \mathrm{k} 2, \mathrm{k} 3$ located on the $\mathrm{IO} / 02$ card remain in non-active state. With such settings, the TBA-ST measures only battery monoblock voltages.

\subsection{Operation with TBA-ST}

If the circuit breaker f.Tba is closed (as shown in Fig. 3b) and the G.TBA signal appears at the input of IO/02 card, then in response to the signal demanding the connection of battery BAT-1, BAT-2 or BAT-3 (e.g. BAT-1 for bat- tery B1) to the TBA-ST unit for testing, the IO/02 card closes k1 relay. As a result (for battery B1), relay RGR-1 disconnects battery B1 from the system bus, and the contactor K31 connects it to the meter. After testing, when the TBA-ST, in the presence of the G.TBA signal switches off the signal demanding battery connection, and the IO/02 card restores the non-active state of the relay $\mathrm{k} 1$. In response, the contactor K31 disconnects the battery from the meter, and RGR-1 connects it to the power system bus. The operation for other batteries is proceed in the same way. In case of meter power supply failure as well as other fault the state of relays $\mathrm{k} 1-\mathrm{k} 3$ and RGR-1-RGR-3 is unchanged. During switching there is no significant current flow in the RGR-1-RGR-3 disconnectors and K31-K33 contactors.

\section{TBA-ST Meter Test Results}

The TBA-ST capacity meter was tested in a circuit contains a power supply, battery simulator, with real batteries as well as power system simulator (Table 1). Column with 
TBA-ST/22 header is related to data for a meter installed in the power system. The "other" column shows values available for all versions. The result of testing the electromagnetic disturbances level generated by the TBA-ST is shown in Fig. 4.

Table 1

The TBA-ST meter parameters

\begin{tabular}{|c|c|c|}
\hline Parameter & TBA-ST/22 & Other \\
\hline Rated voltage battery & \multicolumn{2}{|c|}{$48 \mathrm{~V}$ (54 V power system) } \\
\hline $\begin{array}{l}\text { Operating programmable current } \\
\text { for discharging and charging }\end{array}$ & $2-20 \mathrm{~A}$ & $2-50 \mathrm{~A}$ \\
\hline Number of battery strings & Up to 3 & Up to 6 \\
\hline $\begin{array}{l}\text { Number of controlled } \\
\text { monoblocks of each battery }\end{array}$ & Up to 4 & Up to 24 \\
\hline End of charge criterion & \multicolumn{2}{|c|}{$\begin{array}{c}\text { Capacity consumption as } \\
\text { percent or final } \\
\text { monoblock voltage }\end{array}$} \\
\hline $\begin{array}{l}\text { Accuracy of voltage, current, } \\
\text { charge and time measurement }\end{array}$ & Better than $1 \%$ & \\
\hline $\begin{array}{l}\text { Maximum energy losses on } \\
\text { discharging/charging }\end{array}$ & $5.3 \%$ & \\
\hline $\begin{array}{l}\text { Communication with power } \\
\text { system }\end{array}$ & \multicolumn{2}{|c|}{ DC voltage (Fig. 5) } \\
\hline $\begin{array}{l}\text { Remote management } \\
\text { (Modbus RTU protocol) }\end{array}$ & \multicolumn{2}{|c|}{$\begin{array}{l}\text { RS485 or Ethernet } \\
\text { interface }\end{array}$} \\
\hline
\end{tabular}

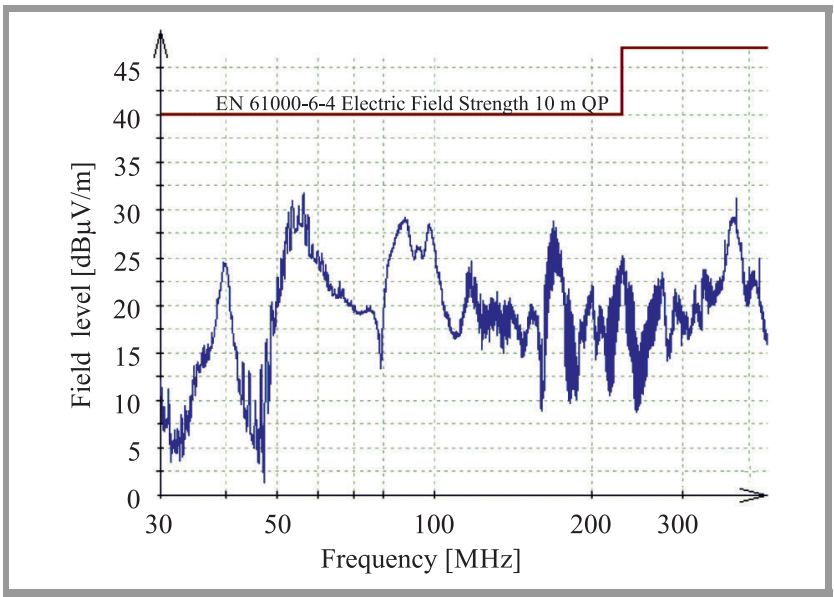

Fig. 4. EMC disturbance level generated by the TBA-ST/22 during battery discharging at rated current.

\section{Power System Testing with TBA-ST Function Disabled}

The scope and testing results of system with the TBAST disabled are presented in Table 2. The column "result" contains reference to the sections with further details.

\subsection{Power System Operation}

The power system indicates operational status and emergency states. The monitoring and power system programming is possible from the signaling module or from a PC
Table 2

Issues to check in the SBE200SL power system

\begin{tabular}{|l|c|}
\hline \multicolumn{1}{|c|}{ Issues to test } & Result \\
\hline \hline Supply voltage 230 V or 3×230/400 V & + \\
\hline Floating voltage 48-56 V & + \\
\hline Automatic charging 53-58 V & + \\
\hline Power system equipment, protection devices & 3.1 \\
\hline Easy access to batteries and fuses & + \\
\hline Signaling and programming & 5.1 \\
\hline Battery disconnection at low voltage & 5.2 \\
\hline Charging after AC mains failure & $5.2-5.3$ \\
\hline Voltage drop in battery circuits & 5.4 \\
\hline TBA-ST function on/off & + \\
\hline
\end{tabular}

via RS232 serial interface or as well as by Ethernet based network and the web server. The available programming includes value of floating voltage, charging current and temperature correction of battery charging voltage, as well as RGR control voltage threshold. Such settings are password protected. In addition, certain options are available only for the maintenance engineers.

\subsection{Battery Disconnection at Mains Failure}

Figure 5 shows the voltage and current waveforms recorded during long-lasting voltage interruption in the $\mathrm{AC}$ mains.

While AC mains failure (AC off), the system bus voltage Us1 and battery voltage Ub lowers, so loads (Io) the current fed with power Ib1 (drawn from the battery) increases. When voltage on the system bus (and the battery voltage) drops to programmed $43 \mathrm{~V}$ value, the controller switches off power supply to the RGR. The RGRs disconnect (RGR off) all batteries from the system bus and DC loads lose power.

When $\mathrm{AC}$ mains is back (AC on), the PS rectifiers power system bus Us2. The controller closes the RGRs (RGR on) and $\mathrm{Ub}$ voltage is fed to the batteries from the system power bus. Initially, the charging is high current power Ib2 and is limited by maximal output current (90 A) and load

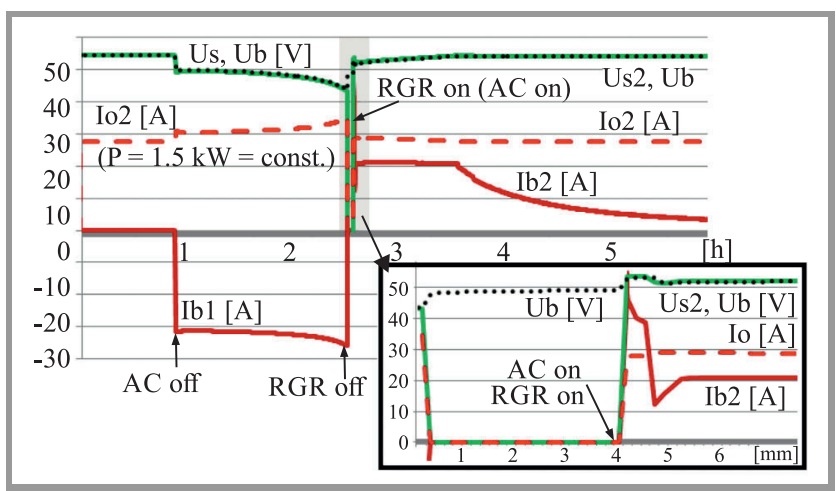

Fig. 5. Long-lasting voltage interruption in the AC mains. (See color pictures online at www.nit.eu/publications/journal-jtit) 
current. Then the rectifiers decrease the voltage, hence battery charging after approximately $1 \mathrm{~min}$ reach programmed 20 A value. While battery is charged, the Ib2 current decreases to the low maintenance level (approx. 0.2 A).

\subsection{Battery Charging After Mains Return}

On short mains voltage interruption, which does not cause the RGRs trip, the battery is charged to the floating voltage with programmed $20 \mathrm{~A}$ value. Next it is charged with decreasing current. An automatic charging feature can be programmed after each AC voltage interruption with a duration exceeding the preset time. The battery will be charged with voltage increased to a programmed value for the entered period. In the tested Benning power supply system, battery charging after mains interruption to floating value $\left(54.4 \mathrm{~V}\right.$ at $\left.+20^{\circ} \mathrm{C}\right)$, and periodic battery charging with boost voltage is performed by the TBA-ST meter.

\subsection{Voltage Drops in Battery Circuits}

A voltage drop in the distribution powerlines should not exceed $0.5 \mathrm{~V}$. The total voltage drop between battery terminals and the load connector located on telecommunication equipment should not exceed 1.2 V.

For load up to $90 \mathrm{~A}$, with both (B1, B2) connected batteries, and disconnected B3 battery while rectifiers are off, the voltage drop in the power system (tested between the system bus ground and the battery negative terminal) is less than $180 \mathrm{mV}$. The voltage drop between the system bus and the battery fuse terminal does not exceed $65 \mathrm{mV}$.

\section{PS Testing with a TBA-ST}

Table 3 presents list of Benning power supply system issues being tested with the TBA-ST, with reference to subsections and detailed description or test result.

Table 3

Power system functions with active TBA-ST meter

\begin{tabular}{|l|c|}
\hline \multicolumn{1}{|c|}{ Issue } & $\begin{array}{c}\text { Result/ } \\
\text { section }\end{array}$ \\
\hline \hline All power system functions maintained & + \\
\hline Installation and replacement of TBA-ST meter & 6.1 \\
\hline Configuring and programming & 6.2 \\
\hline Communication with management system & 6.3 \\
\hline Meter integration with the power system & 3.3 and 6.4 \\
\hline Test initial conditions & 6.5 \\
\hline Remote disconnection of batteries & + \\
\hline Battery capacity measurements & 6.6 \\
\hline $\begin{array}{l}\text { Influence of AC mains voltage interruptions } \\
\text { on measurements }\end{array}$ & 6.7 \\
\hline Remote operation hold and continue & + \\
\hline Battery test by cable with remote readout & 6.8 \\
\hline Tolerance of user faults & + \\
\hline
\end{tabular}

\subsection{Meter Installation and Replacement}

The TBA-ST meter is installed inside the power system enclosure. Together with the ZSN-5 communication controller, it occupies $1 \mathrm{U} \times 200 \mathrm{~mm}$. The instrument is connected to the power system by cable with DB-37 connector. In case the $\mathrm{ZSN}-5$ controller is not used, the communication with the management system (e.g. PC computer) takes place via the internal Ethernet interface module with RJ-45 port. In case of system maintenance, the meter replacement requires only few manual operations: opening of the circuit breaker f.TBA, overcurrent breakers in the battery circuits (F31-33) and power system (F57), 4 screws and DB-37 connector removal. Such maintenance does not influence the power system operation.

\subsection{Configuring and Programming the TBA-ST Unit}

The internal settings as well as operational currents are set by PCB jumpers. The threshold voltages can be software programmed after pressing a button on front panel. The parameters of the batteries being test (i.e. number of cells, storage capacity, block voltage, installation date, discharging and charging currents, charge to be drawn, final charging as well as discharging voltage) can be modified locally from dedicated PC software or remotely through the ZSN-5 controller (password protected). Remote monitoring and battery test initiation are performed in a similar way.

\subsection{Communication with the Host Management System}

The data transmission between the TBA-ST unit and the management system uses Modbus RTU protocol. The TBA-ST meter accepts the following groups of commands of the protocol: $0 \times 03$ i and $0 \times 04$ - read multiple registers; $0 \times 06$ - read single register; $0 \times 10$ - write multiple registers; $0 \times 11$ - report controller ID. Physical communication can use RS232, RS485 or Ethernet (M2M) interfaces. In Table 4 all recognized commands are summarized.

\subsection{Meter Integration with the Power System}

The communication of TBA-ST meter with MCU-based controller is implemented by using IO/02 card using slowly-variable DC signals (Fig. 3). A detailed description of both units' interoperability is provided in Subsection 3.3. The used method of information exchange is presented in Fig. 6. The active state of the signal (high level) G.MCU is "+power system", and for the other ones, feeding the internal reference potential "MS" of card IO/02 to its respective input, which is performed by MOSFET relays in the TBA-ST meter circuit.

\subsection{Test Initial Conditions}

The TBA-ST meter can request any battery bank disconnection from the system bus "-power system" and to switch it on to the meter input when all of the following conditions are fulfilled simultaneously: 
Table 4

Modbus RTE protocol commands used in TBA-ST meter

\begin{tabular}{|c|c|c|}
\hline Description & Type & Address range \\
\hline Configuration parameters & $\mathrm{R} / \mathrm{W}$ & $400-465$ \\
\hline $\begin{array}{l}\text { Values from external } \\
\text { measurements }\end{array}$ & W & $500-524$ \\
\hline TBA-ST current state & $\mathrm{R}$ & 100 \\
\hline Battery monoblock voltages & $\mathrm{R}$ & $110-133$ \\
\hline $\begin{array}{l}\text { Commands concerning B1/2/3 } \\
\text { batteries*) }\end{array}$ & W & $300-302$ \\
\hline Result of current test & $\mathrm{R}$ & $170-179$ \\
\hline Results of last battery test & $\mathrm{R}$ & $600-616$ \\
\hline $\begin{array}{l}\text { Measurement result for each } \\
\text { battery }\end{array}$ & $\mathrm{R}$ & $200-244$ \\
\hline \multicolumn{3}{|c|}{$\begin{array}{l}\text { *) Commands to the meter include: } \\
\text { - battery equalization charging, } \\
\text { - control battery discharge followed by charging return, } \\
\text { - charging equalization followed by discharging and } \\
\text { charging return, } \\
\text { - battery disconnection from power system bus, } \\
\text { - signals test by MCU controller. }\end{array}$} \\
\hline
\end{tabular}

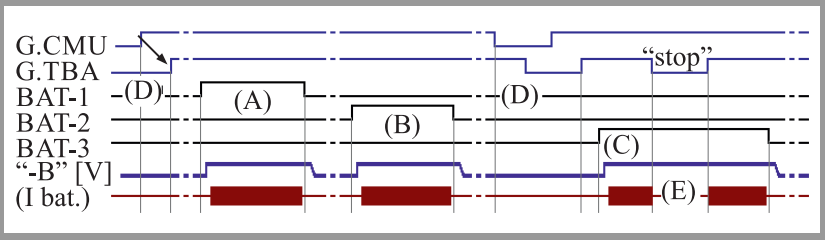

Fig. 6. DC communication method with TBA-ST device.

- it has received the instruction from the supervisory computer,

- the switch "f. TBA-ST" is on,

- it is not in "stop" state,

- all batteries are connected to the system bus, by means their voltage difference is less than $0.2 \mathrm{~V}$.

The TBA-ST will refuse return to non-active state without performing the test if the correct battery voltage (43-57 V) does not appear on its input within 2 minutes from sending the command, or the voltage difference between any 12-voltage blocks of the battery is greater than $1.8 \mathrm{~V}$.

\subsection{Battery Capacity Measurements}

The process of B1 and B2 battery capacity measurement as voltage waveforms for each block is shown in Fig. 7. One can see, that no AC mains interruption occurred during the test. Discharging was performed by 10 A current (10 hour, $0.1 \mathrm{CA}$ ) until the $10.80 \mathrm{~V}$ voltage is reached on the B1 battery case, or the declared charge of $80 \mathrm{Ah}(80 \% \mathrm{Q})$ has been consumed - battery B2 case. After discharging, the batteries were immediately charged back with 10 A current to $56.5 \mathrm{~V}$ end voltage.
During testing battery block voltage measurement errors were below $0.5 \%$, and discharging current fluctuations and its measurement error was under $1 \%$. The instrument showed that battery B1 has a 52 Ah capacity, and battery B is over $80 \mathrm{Ah}$. Interestingly, after $4 \mathrm{~h}$ of discharging, the worst block $(\mathrm{C}=52 \% \mathrm{Q})$ of the bad $\mathrm{B} 1$ had a higher voltage than the best B2 $(\mathrm{C}>80 \% \mathrm{Q})$, although both batteries are of the same type, and have been used in a similar bad condition for 3 years.

During charging, when battery voltage reaches the system bus voltage, a voltage and current swing occurred (see 3 in Fig. 7). It is a result of voltage drop on the battery (HZB12-100FA) during switching internal converter from buck to boost mode. This effect does not influence on battery charging level, the charge delivered to the battery or the battery charging time.

\subsection{Influence of AC Mains Voltage Interruptions on Capacity Measurements}

A mains voltage interruption can occur after battery bank disconnection from the power system. The voltage and current diagrams for such case are shown in Fig. 8. The used notation is: 1 - voltage of battery being charged, 2 voltage of battery being discharged, 3 - voltage of system bus, 4 - voltage of loads with constant power demand of $1500 \mathrm{~W}, 5 \mathrm{a}$ - current of battery being charged, $5 \mathrm{~b}$ - current of battery being discharged, $5 \mathrm{c}$ - battery current during backup, 6 - battery voltage during backup, 7 - moment of switching to battery backup mode, 8 - current of other batteries connected to system bus "-power system".

Figure 8a shows the effect of long-lasting AC mains voltage interruption during the discharging operation, while Fig. 8b shows the same case during equalization B2 battery charging. When voltage on the system bus drops below $51 \mathrm{~V}$, charging is stopped but discharging is continued. While voltage on the system bus lowers below 48.5 $\mathrm{V}$, the meter switches into backup mode "7" in Fig. 8b. The meter circuit contains power converter with current limiter. It draws energy from the battery being tested and transfers it to power system bus. While AC voltage appears, the battery being tested is recharged and connected to the system after its voltage and system bus becomes equal. This operation may few repetition, as V-I characteristic varies in time from varies programmed values. Battery current is not constant, and the Ah drawn may be greater than preset.

The effect of short AC mains voltage interruption while B1 battery is tested, in case the voltage on the system bus exceed $48.5 \mathrm{~V}$ threshold, is illustrated in Fig. 8c. During battery charging, the meter stops the process for total power failure period. If voltage interruption occurs during battery discharging, the meter continues operation with uncharged current. Due to described purpose of operation the measurements are fully reliable - the measured available capacity of batteries for current of $0.2 \mathrm{CA}=20 \mathrm{~A}$ was $46 \mathrm{Ah}\left(\mathrm{C}_{5}=46 \mathrm{Ah}\right)$ or $2.22 \mathrm{kWh}$, which corresponds to $50 \mathrm{Ah}$ (for $0.1 \mathrm{CA}$ ), and during return charging, a charge 

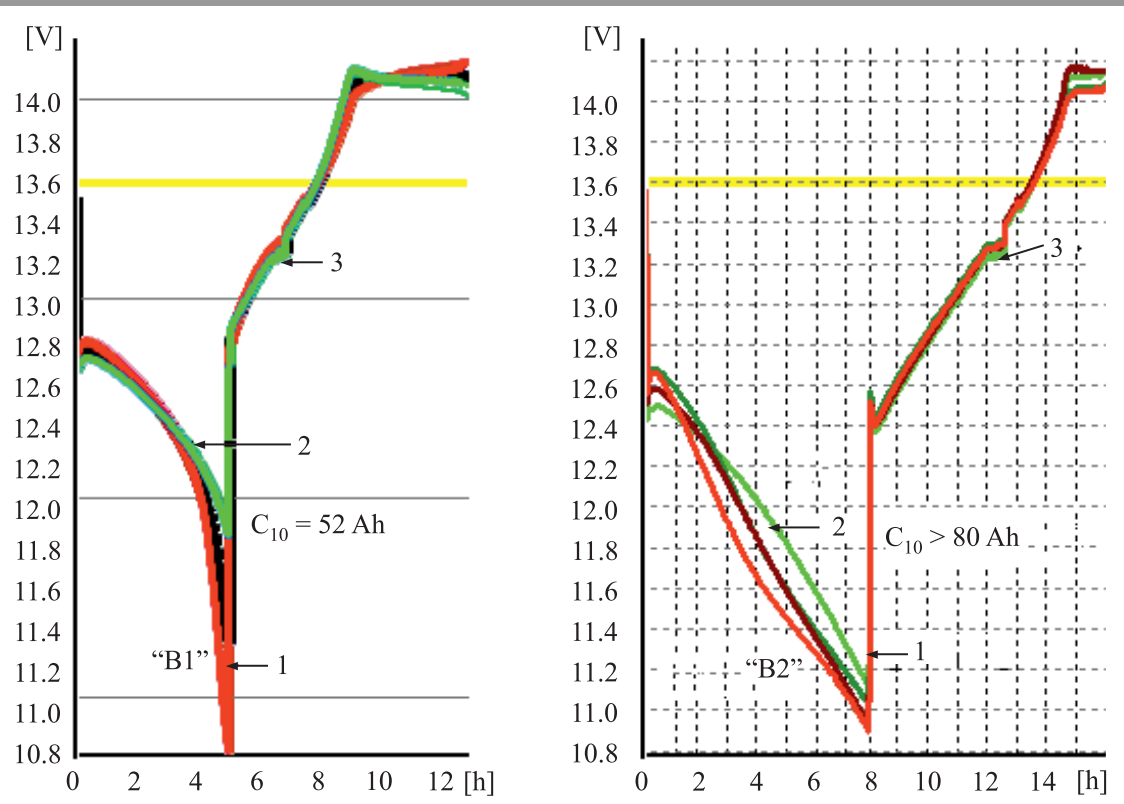

Fig. 7. Voltages during battery test process on different block: 1 - worst block, 2 - best block, 3 - fluctuations during charging.

(a)
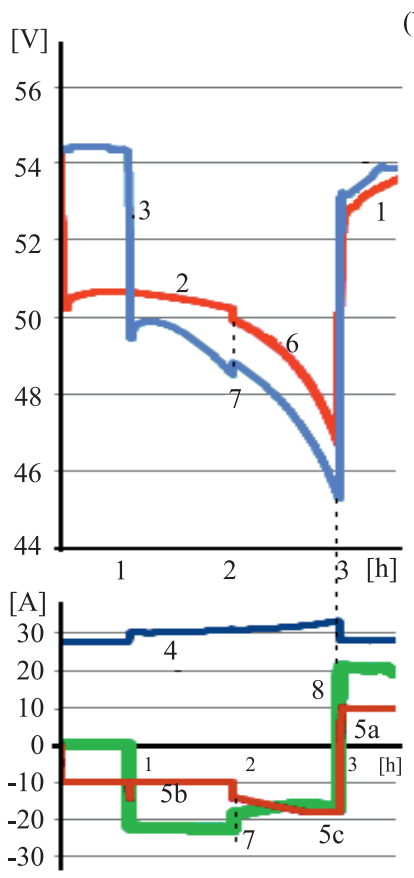

(b)
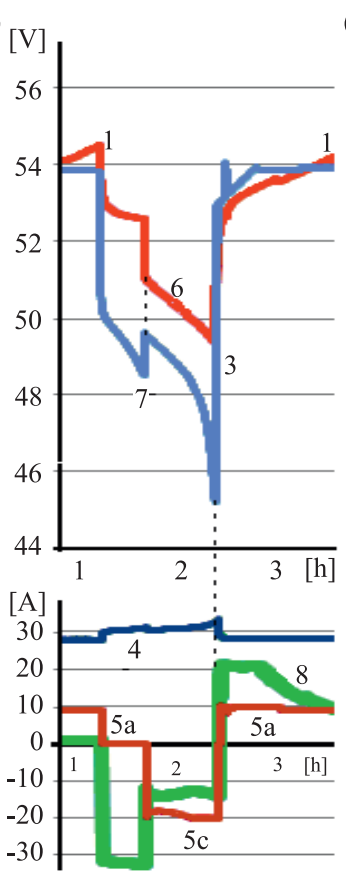

(c)
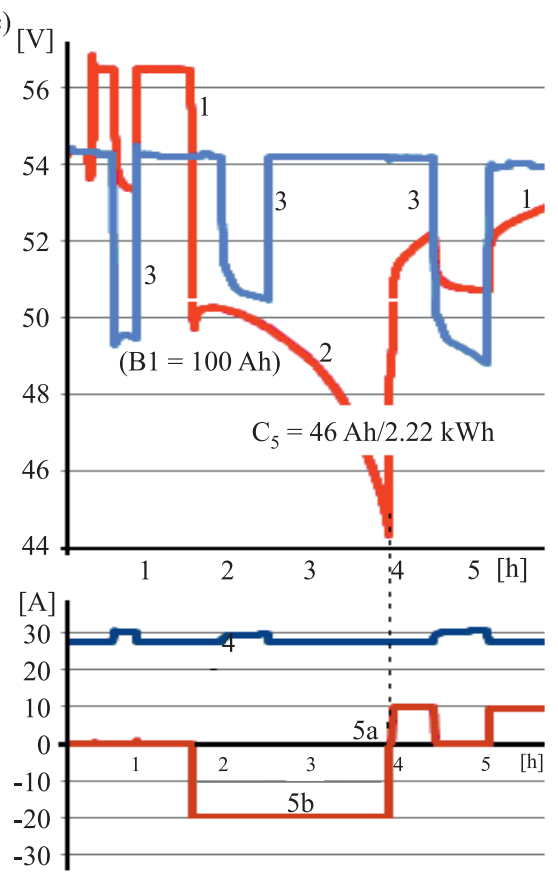

Fig. 8. Battery voltage and current at interruption in phase of: (a) discharging, (b) equalization charging, (c) full testing.

of $54 \mathrm{Ah}$ was delivered to the batteries (about $10 \%$ greater than drawn).

\subsection{Remote Readout of Battery Test Results}

The testing results can be downloaded to remote computer by the ZSN-5 communication controller or (alternatively) via built-in Ethernet interface. For testing purposes and at the system commissioning stage, the results can be by using of the TBA_Starter and TBA_Reporter programs (Fig. 9).

\section{Conclusions}

The results of testing the SBE200SL power supply system with a built-in TBA-ST meter showed that it can be used for powering telecommunications devices at maintenance-free 


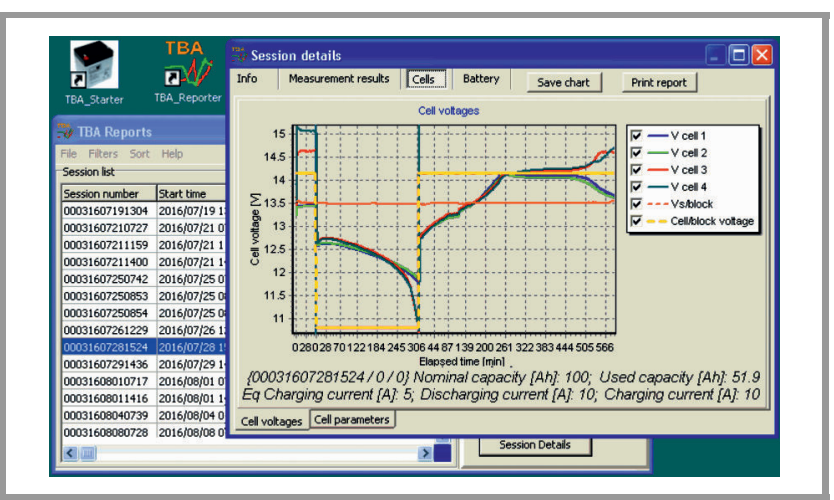

Fig. 9. Battery test results as represented in the TBA_Reporter software.

sites. A minor upgrade of a typical power supply system provides the capability to measure the available capacity of individual battery banks and remote management.

The tested SBE200SL power supply system can be additionally equipped, with a second set of rectifiers, which increases the efficiency. In order to extend the time of energy autonomy, without changing power system components, three $100 \mathrm{Ah}$ batteries in an external rack housing could be exchanged with a higher capacity ones. The power system could also be additionally provided, without significant changes in equipment and functioning, with a fourth RGR disconnector switch and battery contactor, which will make it possible to individually test the available capacity of four battery strings (the TBA-ST meter can control up to 6 batteries of 1,000 Ah each).

\section{References}

[1] R. Kobus, P. Kliś, and P. Godlewski, "Maintenance of lead-acid batteries used in telecommunications systems", J. of Telecommun. and Inform. Technol., no. 4, pp. 106-113, 2015.

[2] P. Godlewski et al., "Method and system for remote measurement of available capacity of the batteries in the telecommunications power system”, Patent PL 219471, Patent Office of the Republic of Poland, 2015 (in Polish).

[3] "Telecommunications Power System $48 \mathrm{~V}$ type SBE300SL, SBE200SL - O\&M Manual”, version R-60704635-4, Benning Power Electronics (in Polish).

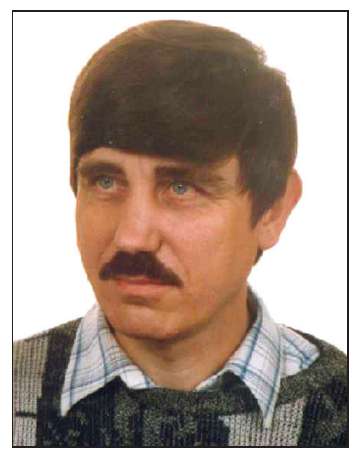

Paweł Godlewski received his B.Sc. degree from the Faculty of Electronics of the Warsaw University of Technology in 1973. He has been working at the National Institute of Telecommunications since 1973. He is the designer of many devices, and co-author of a system for the assessment of quality of telecommunications services, a well as AWP-IL and TBA-IL ATE equipment. $\mathrm{He}$ is the author of numerous scientific publications and co-author of patented solutions. His research interests include: visualization systems used in the telecommunications sector, programmable measurement devices for telecommunications.

E-mail: P.Godlewski@itl.waw.pl

National Institute of Telecommunications

Szachowa st 1

04-894 Warsaw, Poland

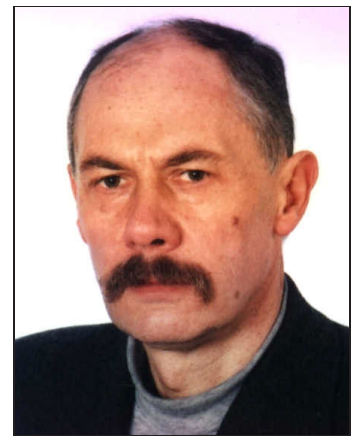

Ryszard Kobus received his B.Sc. and M.Sc. degrees from the Faculty of Electronics of the Warsaw University of Technology in 1975. Mr. Kobus has been working at the National Institute of Telecommunications since 1975 . He is a member of the Expert Technical Committee CEN/TC 331 specializing in postal services, and the deputy chairman of the Postal Service Committee PKN/TC 259. He is a co-author of many patented telecommunications solutions. His research interests include: telecommunications, measurements and evaluation of quality of telecommunications services, quality surveys, evaluation the quality of postal services, standardization.

E-mail: R.Kobus@itl.waw.pl

National Institute of Telecommunications

Szachowa st 1

04-894 Warsaw, Poland

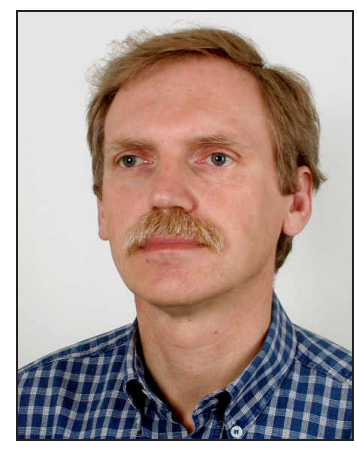

Paweł Kliś received his B.Sc. degree from the Faculty of Electrical Engineering of the Opole School of Engineering in 1976. He has been working at the National Institute of Telecommunications since 1976, formerly in the Power Systems Department, currently in the Central Chamber for Telecommunications Metrology. He is a co-designer of numerous telecommunications power systems and devices. He is a co-author of several scientific publications and co-author of several patents. His research interests include: telecommunications power systems, metrology of basic electrical parameters.

E-mail: P.Klis@itl.waw.pl

National Institute of Telecommunications

Szachowa st 1

04-894 Warsaw, Poland 